\title{
Efecto protector en cirrosis hepática inducida en ratas del extracto etanólico de las hojas de Piper aduncum comparado con silimarina
}

\author{
Piper aduncum leaves ethanol extracts protective effect compared with
} silymarin in liver cirrhosis induced in rats

\author{
Jorge Arroyo ${ }^{1,2}$, Yuan Almora ${ }^{4,5}$, Mariano Quino ${ }^{1,3}$, Ernesto Raez ${ }^{5}$, Jaime Martínez ${ }^{6}$, \\ Jesús Buendia ${ }^{7}$, Deybis Baca ${ }^{7}$, Renan Hañari \\ Instituto de Investigaciones Clinicas, Facultad de Medicina, Universidad Nacional Mayor de San Marcos, Lima, Perú. \\ ${ }^{2}$ Laboratorio de Farmacología, Facultad de Medicina, Universidad Nacional Mayor de San Marcos, Lima, Perú. \\ ${ }^{3}$ Gastroenterología, Hospital Nacional Dos De Mayo \\ ${ }^{4}$ Farmacia, Hospital Nacional Hipólito Unanue. \\ ${ }^{5}$ Instituto de Patología de la Facultad de Medicina, Universidad Nacional Mayor de San Marcos, Lima, Perú. \\ ${ }^{6}$ Seguro Social de Salud (EsSalud), Tingo Maria, Huánuco, Perú. \\ ${ }^{7}$ Unidad de Posgrado, Facultad de Farmacia y Bioquímica, Universidad Nacional Mayor de San Marcos, Lima, Perú.
}

\begin{abstract}
Resumen
Objetivos: Evaluar la eficacia protectora del extracto etanólico de hojas de Piper aduncum (matico) y su fitomedicamento en cápsulas, en la cirrosis hepática inducida en ratas. Diseño: Experimental. Lugar: Facultad Medicina, Universidad Nacional Mayor de San Marcos, Lima, Perú. Material biológico: Hojas de Piper aduncum, y Rattus norvegicus, cepa Holtzman. Intervenciones: Las hojas fueron recolectadas en el distrito de Huariaca, departamento de Pasco. El fitomedicamento en cápsulas se preparó a partir del extracto etanólico de la planta. La cirrosis fue inducida con fenobarbital $0,5 \mathrm{mg} / \mathrm{mL}$, diluida en el agua de beber por 15 días, y luego, tetracloruro de carbono $0,2 \mathrm{~mL} / \mathrm{kg}$ en aceite de oliva 1:1, oralmente por 7 dias. Se colectó una muestra de sangre para determinar perfil hepático y malondialdehido; los animales fueron sacrificados extrayéndose el hígado para estudio histopatológico. Los datos fueron evaluados mediante técnicas multivariadas, con valor $p<0,05$. Principales medidas de resultados: Grado de lesión hepática, marcadores bioquímicos, estrés oxidativo. Resultados: El extracto y el fitomedicamento a $200 \mathrm{mg} / \mathrm{kg}$ disminuyeron los valores de TGP $(p<0,621)$, bilirrubina total $(p<0,385)$ y bilirrubina directa $(p<0,283)$ e incrementaron las proteinas totales $(p<0,539)$ y albúmina $(p<0,114)$, similar al grupo silimarina. El colágeno, la fibrosis y el nivel de daño hepático se vieron aumentados con tetracloruro de carbono; estos indicadores se redujeron con los diferentes tratamientos y la silimarina. El marcador de estrés oxidativo se redujo con los tratamientos aplicados ( $\mathrm{p}$ 0,002). Conclusiones: El extracto etanólico de las hojas de Piper aduncum (matico) y su fitomedicamento ejercieron efecto protector de la cirrosis inducida en ratas, comparativamente con la silimarina.
\end{abstract}

Palabras clave: Matico, cirrosis hepática experimental, extractos vegetales, ratas Sprague-Dawley.

Abstract

Objectives: To determine the protective effect of Piper aduncum (matico) leaves ethanol extract and its phytomedicine in capsules in liver cirrhosis induced in rats. Design: Experimental. Location: Faculty of Medicine, Universidad Nacional Mayor de San Marcos, Lima, Peru. Biological material: Leaves of Piper aduncum, and Rattus norvegicus, Holtzman strain. Interventions: The leaves were collected in La Merced district, Pasco department. The phytomedicine capsules were prepared from the plant ethanol extract. Cirrhosis was induced with phenobarbital $0.5 \mathrm{mg} / \mathrm{mL}$ diluted in drinking water during 15 days, then carbon tetrachloride $0.2 \mathrm{~mL} / \mathrm{kg} 1: 1$ in olive oil orally during 7 days. Blood samples were drawn in order to determine liver profile and malondialdehyde; the animals were sacrificed extracting the liver for pathology study. Data were evaluated by multivariate techniques with $p<0.05$. Main outcome measures: Degree of liver injury, biochemical marks, oxidative stress. Results: About $200 \mathrm{mg} / \mathrm{kg}$ of both extract and phytomedicine decreased ALT values $(p<0.621)$, total bilirrubin $(p<0.385)$ and direct bilirrubin $(p<0.283)$ and increased total protein $(p<0.539)$ and albumin $(p<0.114)$, similar to silymarin group. Collagen, fibrosis and liver damage degree increased with carbon tetrachloride, and reduced with the different treatments and silymarin. Oxidative stress marker was also reduced with the applied treatments $(p<0.002)$. Conclusions: Piper aduncum (matico) leaves ethanol extract and its phytomedicine had protective effect of cirrhosis induced in rats similar to silymarin.

Key words: Matico, liver cirrosis/experimetal, plant extracts, rats/Sprague-Dawley.

An Fac med. 2012;73(2):85-91 


\section{INTRODUCCIÓN}

La cirrosis hepática es uno de los principales problemas de salud en el mundo, debido a su alta morbilidad y mortalidad. Las tasas de defunción más elevadas se registran en Moldavia (91 por 100000 habitantes) y Hungría (85 por 100 000), mientras que las cifras más bajas, entre 3 y 5 por 100000 habitantes, corresponden a Irlanda, Colombia, Holanda, Singapur, Israel y Noruega. En algunos países de América Latina, como Chile y México, la cirrosis hepática ocupa entre el 50 y 60 lugar como causa de muerte general. En un estudio de carga enfermedad en el Perú, en el año 2004, los años de vida saludables perdidos (AVISA) por cirrosis fue de 76510 años entre hombres y mujeres de personas mayores de 15 años. De estos años de vida saludables perdidos, 15023 fueron por años vividos con discapacidad (AVD) y 61487 por años de vida perdidos por muerte prematura (AVP) ${ }^{(1)}$.

Al desarrollarse la fibrosis, y en particular la cirrosis, se asocia con una significativa morbilidad y mortalidad, lo que conlleva a constante investigación sobre el desarrollo de estrategias de compuestos con propiedades antifibróticas ${ }^{(2)}$. El tratamiento farmacológico de la cirrosis busca detener o retrasar su progreso de daño de las células hepáticas y reducir las complicaciones; los fármacos pueden inducir efectos no esperados, por lo que se hace necesaria la búsqueda de compuestos con mínimos efectos adversos.

Al Piper aduncum, popularmente conocido como matico, se le atribuyen propiedades medicinales como antiséptico y cicatrizante de heridas ${ }^{(3)}$; usado en problemas intestinales, erisipelas, desordenes hepáticos ${ }^{(4)}$, contiene flavonoides y compuestos fenólicos, y estos metabolitos le confieren efecto antioxidante y protector del hígado frente a los agentes tóxicos ${ }^{(5-8)}$. También contiene taninos, los que poseen propiedades similares a la de los flavonoides sobre los radicales libres de oxígeno, y por consiguiente actividad hepato- protectora ${ }^{(9)}$. Por ello, el objetivo del presente estudio ha sido determinar el efecto protector del extracto etanólico de las hojas de Piper aduncum (matico) sobre la cirrosis hepática inducida en ratas, así como comparar su efecto con el de la silimarina.

Con este trabajo de investigación, se desea demostrar la eficacia protectora de las hojas de Piper aduncum (HPA), al mejorar el perfil hepático con la administración del extracto etanólico (EEPA) y su fitomedicamento en cápsulas (FCPA), comparado con silimarina en ratas con inducción de cirrosis hepática; explicar el posible mecanismo de acción hepatoprotector del EEPA en ratas con inducción de cirrosis hepática, determinando el nivel de estrés oxidativo y depósito de colágeno en el parénquima hepático; analizar los cambios anatomopatológicos del tejido hepático en ratas con inducción de cirrosis hepática que recibieron EEPA dado por vía oral y el FCPA; y observar la seguridad del efecto protector del EEPA y su fitomedicamento.

\section{MÉTODOS}

La muestra estuvo constituida por 70 ratas Holtzman machos, con peso corporal de $220 \pm 20 \mathrm{~g}$. Aleatoriamente fueron distribuidas en 7 grupos de 10 animales cada grupo, según el siguiente diseño experimental: 1) normal, solvente 3\% de solución de polisorbato de sodio, $4 \mathrm{~mL} / \mathrm{kg}$; 2) fenobarbital $200 \mathrm{mg}$ en agua de beber, por 15 días; luego, por 7 días tetracloruro de carbono de 0,2 $\mathrm{mL} / \mathrm{kg}$ (diluido al $50 \%$ en aceite de oliva), relación $1: 1(\mathrm{~T})$; 3) $\mathrm{T}+$ silimarina $25 \mathrm{mg} / \mathrm{kg}$; 4) $\mathrm{T}+$ extracto etanólico $100 \mathrm{mg} / \mathrm{kg}$; 5) $\mathrm{T}+$ extracto etanólico $200 \mathrm{mg} / \mathrm{kg}$; 6) $\mathrm{T}+$ extracto etanólico $400 \mathrm{mg} / \mathrm{kg}$; y, 7) $\mathrm{T}+$ fitomedicamento conteniendo extracto etanólico, 200 $\mathrm{mg} / \mathrm{kg}$.

Para la preparación del extracto etanólico de Piper aduncum ${ }^{(10,11)}$, las hojas de la planta fueron recolectadas en el mes de marzo del año 2011, en el distrito de Huariaca, provincia de Pasco, departamento de Pasco. La planta fue colocada bajo sombra, con buena ventilación, secada, pulverizada y macerada con etanol $70^{\circ}$ por 7 días; luego se la decantó, filtró, evaporó el solvente, para obtener el extracto etanólico. Al extracto se le realizó el estudio de identificación cualitativa de metabolitos secundarios.

Las cápsulas del extracto etanólico de las hojas de Piper aduncum fueron manufacturadas según lo expuesto en Fauli $1993^{(12)}$. Para ello se verificó la densidad del extracto, se añadió Veegum Hv a la solución resultante y luego se agregó el Aerosil 200, agitándose vigorosamente hasta la completa homogenización; se llevó a sequedad en estufa a temperatura de $45^{\circ} \mathrm{C}$.

La inducción de la lesión hepática se desarrolló mediante un modelo experimental utilizando fenobarbital más tetracloruro de carbono ${ }^{(13)}$. Se utilizó 70 ratas machos cepa Holtzman, con peso corporal promedio de $220 \pm 20 \mathrm{~g}$. Para inducir las lesiones hepáticas crónicas, en un inicio se usó el fenobarbital en agua de beber, durante 15 días, para luego administrar el tetracloruro de carbono $\left(\mathrm{CCl}_{4}\right)$. El fenobarbital fue solubilizado en el agua de beber en una concentración de $0,5 \mathrm{~g} / \mathrm{L}$; el consumo de agua fue a libertad, por un tiempo de dos semanas; luego, se le administró tetracloruro de carbono $\left(\mathrm{CCl}_{4}\right)$ en dosis de $0,2 \mathrm{~mL} / \mathrm{kg}$ (diluido al $50 \%$ en aceite de oliva), relación 1:1; se le administró por vía oral durante siete días.

Para la obtención de la muestra, se anestesió a los animales y se obtuvo aproximadamente $5 \mathrm{~mL}$ de sangre mediante punción cardiaca. Las variables de estudio fueron: 1) perfil hepático, 2) marcador de estrés oxidativo, y, 3) estudio histopatológico.

En sangre se determinó el perfil hepático (bilirrubina total, bilirrubina directa, proteínas totales, albúmina, transaminasa glutámico-oxalacética (TGO) y fosfatasa alcalina).

La determinación del marcador de estrés oxidativo fue realizada en suero procedente de la sangre extraída de las 
Tabla 1. Ensayo fitoquímico del extracto etanólico de las hojas de Piper aduncum (matico).

\begin{tabular}{ccc} 
Reacciones & Metabolito secundario & Cantidad \\
Dragendorff & Alcaloides & ++ \\
Mayer & Alcaloides & ++ \\
Shinoda & Flavonoides & +++ \\
Índice de espuma & Saponinas & ++ \\
Cloruro férrico & Compuestos fenólicos & ++ \\
Olor & Aceites & ++ \\
Gelatina & Taninos & ++ \\
Liebermann & Esteroides & - \\
Ninhidrina & Aminoácidos & + \\
Bortranger & Quinonas & + \\
\hline
\end{tabular}

$(-)$ Ausencia; $(+)$ = Poca cantidad; $(++)=$ Regular cantidad; $(+++)$ = Bastante cantidad ratas sometidas a inducción de cirrosis hepática. Se efectuó la prueba de lipoperoxidación, que implica la conversión oxidativa de ácidos grasos insaturados (hidroperóxidos lipídicos o lipoperóxidos). La lipoperoxidación fue medida mediante la producción de malondialdehído, que al reaccionar con el ácido tiobarbitúrico forma un complejo coloreado que fue leído a $535 \mathrm{~nm}$; se usó el coeficiente de extinción molar ${ }^{(14)}$ de MDA-TBA: $1,56 \times 10^{5} \mathrm{M}^{-1} \mathrm{~cm}^{-1}$.

La seguridad del efecto protector del extracto etanólico de hojas de Piper aduncum y su fitomedicamento fue evaluada al observar los cambios del peso corporal e indicadores bioquímicos: colesterol total, colesterol HDL, triglicéridos, glucosa y urea; así como a nivel de los glóbulos blancos.

Una vez sacrificados los animales al administrar pentobarbital $100 \mathrm{mg} / \mathrm{kg}$, se procedió a realizar cortes de hígado: $0,5 \times 1,0 \mathrm{~cm}$ de espesor, los que fueron fijados en formol neutro al $10 \%$, siendo seccionados para inclusión en porciones de $2 \mathrm{~mm}$ de espesor; posteriormente, se efectuó cortes con micrótomo, en un espesor de 3 a 5 micras, para luego ser coloreados con HE (hematoxilinaeosina) y revisados con microscopio óptico ${ }^{(15)}$, siendo los indicadores fibrosis, colágeno y daño hepático, cuyas unidades de medida fueron $(-)=$ ausente, $(+)=$ leve, $(++)=$ moderado, $(+++)=$ severo.
El método empleado para realizar comparaciones entre los distintos tratamientos fue el análisis de varianza para un factor. La variable tratamiento tuvo siete categorías (normal, fenobarbitaltetracloruro, T-silimarina $25 \mathrm{mg}, \mathrm{T}$ extracto $100 \mathrm{mg}$, T-extracto $200 \mathrm{mg}, \mathrm{T}$ extracto $400 \mathrm{mg}$, T- fitomedicamento 200 mg. Se realizó la prueba de análisis de varianza con cada una de las variables respuesta, medidas en escala métrica: peso, colesterol, colesterol HDL, triglicéridos, glucosa, urea, transaminasa glutámico-pirúvico (TGP), fosfatasa, abastonados, segmentados, eosinófilos, basófilos, monocitos, leucocitos, linfocitos, bilirrubina total, bilirrubina directa, albúmina y proteína total. El objetivo con cada modelo fue probar el efecto del tratamiento sobre las variables respuesta de manera individual.

\section{RESULTADOS}

En la tabla 1 se observa que el extracto etanólico de Piper aduncum (matico) tuvo mayor cantidad de flavonoides, seguido por compuestos fenólicos y alcaloides. Se obtuvo cápsulas en promedio de $180 \mathrm{mg}$ de extracto en una cápsula, de capacidad de $750 \mathrm{mg}$ en promedio, realizándosele control de calidad fitoquímico y microbiológico.

En la tabla 2 se muestra los cambios sobre el perfil hepático, donde se aprecia que el fenobarbital-tetracloruro de carbono indujo incremento de bilirrubina total y directa, asimismo aumento de transaminasa TGP y fosfatasa alcalina; también disminución de proteínas totales y albúmina; y los tratamientos con silimarina, extracto etanólico y su fitomedicamento mejoraron estos desniveles. El fenobarbital-tetracloruro de carbono aumentó el colesterol total, triglicéridos, glucosa y urea y redujo la HDL, mientras que silimarina, extracto etanólico y su fitomedicamentos no contribuyeron a mayores cambios; en tanto, no se apreció cambios significativos con los glóbulos blancos.

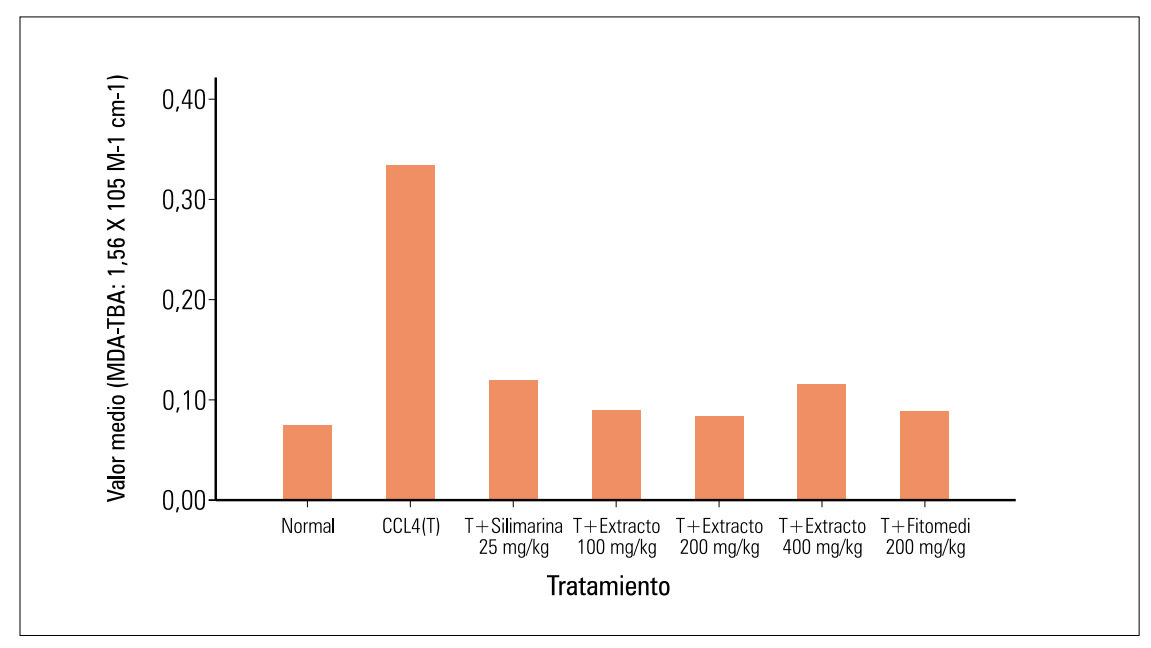

Figura 1. Marcador de estrés oxidativo en suero de ratas. 
Tabla 2. Valores medios y porcentajes de variación del perfil hepático en ratas.

\begin{tabular}{|c|c|c|c|c|}
\hline Variable & Tratamiento & Media & EE & $\% \operatorname{Var}$ \\
\hline Bilirrubina total & Normal & 0,8 & 0,1 & 0,0 \\
\hline \multirow[t]{6}{*}{$p<0,385$} & Tetracloruro (T) & 1,2 & 0,2 & 46,2 \\
\hline & $\mathrm{T}+$ Silimarina $25 \mathrm{mg} / \mathrm{kg}$ & 1,1 & 0,2 & 43,9 \\
\hline & $\mathrm{T}+$ Extracto $100 \mathrm{mg} / \mathrm{kg}$ & 1,1 & 0,1 & 36,9 \\
\hline & $\mathrm{T}+$ Extracto $200 \mathrm{mg} / \mathrm{kg}$ & 1,0 & 0,1 & 24,8 \\
\hline & $\mathrm{T}+$ Extracto $400 \mathrm{mg} / \mathrm{kg}$ & 1,1 & 0,1 & 40,9 \\
\hline & $\mathrm{T}+$ Fitomedi $200 \mathrm{mg} / \mathrm{kg}$ & 1,0 & 0,0 & 31,6 \\
\hline Bilirrubina directa & Normal & 0,4 & 0,1 & 0,0 \\
\hline \multirow[t]{6}{*}{$p<0,283$} & Tetracloruro (T) & 0,7 & 0,1 & 59,9 \\
\hline & $\mathrm{T}+$ Silimarina $25 \mathrm{mg} / \mathrm{kg}$ & 0,6 & 0,1 & 27,1 \\
\hline & $\mathrm{T}+$ Extracto $100 \mathrm{mg} / \mathrm{kg}$ & 0,7 & 0,1 & 56,2 \\
\hline & $\mathrm{T}+$ Extracto $200 \mathrm{mg} / \mathrm{kg}$ & 0,6 & 0,1 & 27,7 \\
\hline & $\mathrm{T}+$ Extracto $400 \mathrm{mg} / \mathrm{kg}$ & 0,7 & 0,1 & 50,8 \\
\hline & $\mathrm{T}+$ Fitomedi $200 \mathrm{mg} / \mathrm{kg}$ & 0,6 & 0,1 & 34,2 \\
\hline Proteínas totales & Normal & 5,7 & 0,3 & 0,0 \\
\hline \multirow[t]{6}{*}{$p<0,539$} & Tetracloruro (T) & 5,4 & 0,1 & $-5,1$ \\
\hline & $\mathrm{T}+$ Silimarina $25 \mathrm{mg} / \mathrm{kg}$ & 5,9 & 0,2 & 2,5 \\
\hline & $\mathrm{T}+$ Extracto $100 \mathrm{mg} / \mathrm{kg}$ & 5,8 & 0,4 & 2,2 \\
\hline & $\mathrm{T}+$ Extracto $200 \mathrm{mg} / \mathrm{kg}$ & 6,0 & 0,2 & 5,7 \\
\hline & $\mathrm{T}+$ Extracto $400 \mathrm{mg} / \mathrm{kg}$ & 5,7 & 0,1 & $-0,5$ \\
\hline & $\mathrm{T}+$ Fitomedi $200 \mathrm{mg} / \mathrm{kg}$ & 6,2 & 0,2 & 8,3 \\
\hline Albúmina & Normal & 3,3 & 0,2 & 0,0 \\
\hline \multirow[t]{6}{*}{$p<0,114$} & Tetracloruro (T) & 3,1 & 0,0 & $-6,7$ \\
\hline & $\mathrm{T}+$ Silimarina $25 \mathrm{mg} / \mathrm{kg}$ & 3,5 & 0,3 & 7,4 \\
\hline & $\mathrm{T}+$ Extracto $100 \mathrm{mg} / \mathrm{kg}$ & 3,9 & 0,3 & 17,5 \\
\hline & $\mathrm{T}+$ Extracto $200 \mathrm{mg} / \mathrm{kg}$ & 3,4 & 0,2 & 3,0 \\
\hline & $\mathrm{T}+$ Extracto $400 \mathrm{mg} / \mathrm{kg}$ & 3,7 & 0,1 & 10,9 \\
\hline & $\mathrm{T}+$ Fitomedi $200 \mathrm{mg} / \mathrm{kg}$ & 3,4 & 0,2 & 1,6 \\
\hline TGP & Normal & 20,5 & 4,1 & 0,0 \\
\hline \multirow[t]{6}{*}{$p<0,621$} & Tetracloruro (T) & 27,6 & 4,5 & 34,8 \\
\hline & $\mathrm{T}+$ Silimarina $25 \mathrm{mg} / \mathrm{kg}$ & 10,8 & 0,8 & $-47,6$ \\
\hline & $\mathrm{T}+$ Extracto $100 \mathrm{mg} / \mathrm{kg}$ & 11,4 & 1,6 & $-44,5$ \\
\hline & $\mathrm{T}+$ Extracto $200 \mathrm{mg} / \mathrm{kg}$ & 9,8 & 1,1 & $-52,4$ \\
\hline & $\mathrm{T}+$ Extracto $400 \mathrm{mg} / \mathrm{kg}$ & 18,5 & 1,8 & $-9,8$ \\
\hline & $\mathrm{T}+$ Fitomedi $200 \mathrm{mg} / \mathrm{kg}$ & 10,9 & 1,0 & $-47,0$ \\
\hline Fosfatasa alcalina & Normal & 120,3 & 13,9 & 0,0 \\
\hline \multirow[t]{6}{*}{$p<0,078$} & Tetracloruro (T) & 153,0 & 9,6 & 27,2 \\
\hline & $\mathrm{T}+$ Silimarina $25 \mathrm{mg} / \mathrm{kg}$ & 110,6 & 6,7 & $-8,0$ \\
\hline & $\mathrm{T}+$ Extracto $100 \mathrm{mg} / \mathrm{kg}$ & 132,1 & 5,2 & 9,9 \\
\hline & $\mathrm{T}+$ Extracto $200 \mathrm{mg} / \mathrm{kg}$ & 138,5 & 12,9 & 15,2 \\
\hline & $\mathrm{T}+$ Extracto $400 \mathrm{mg} / \mathrm{kg}$ & 136,4 & 9,3 & 13,4 \\
\hline & $\mathrm{T}+$ Fitomedi $200 \mathrm{mg} / \mathrm{kg}$ & 126,0 & 10,6 & 4,8 \\
\hline
\end{tabular}

Porcentaje $(\%)$ de variación $=[($ Valor medio del tratamiento X 100)/ Valor medio del normal $)-100)]$. $\mathrm{EE}=$ error estándar; Fitomedi $=$ fitomedicamento.
El colágeno, la fibrosis y el nivel de daño hepático aumentaron con fenobarbital-tetracloruro de carbono; estos indicadores se redujeron con los diferentes tratamientos e incluso silimarina.

El marcador de estrés oxidativo se incrementó con tetracloruro de carbono y redujo con los tratamientos aplicados a un nivel de significancia $\mathrm{p}<0,002$ (figura 1).

El extracto y el fitomedicamento a $200 \mathrm{mg} / \mathrm{kg}$ fueron los que más redujeron los valores de TGP, bilirrubina total y directa, con incremento de proteínas totales y albúmina, cercanos al efecto de silimarina.

\section{DISCUSIÓN}

La cirrosis hepática se asocia frecuentemente con complicaciones tales como ascitis, hipertensión portal, encefalopatía, hemorragia digestiva, e insuficiencia hepatocelular; así como también, infecciones bacterianas ${ }^{(16)}$. En la presente investigación se ha observado la presencia de cirrosis hepática, la misma que fue inducida por la administración de fenobarbital y tetracloruro de carbono (figura 2).

La cirrosis observada se explicaría porque el fenobarbital es un potente estimulante hepático que in vivo produce hipertrofia e hiperplasia a este nivel; del mismo modo, otros carcinógenos celulares frecuentemente son usados en este tipo de modelos experimentales. Asimismo, se ha comunicado que el tratamiento a largo plazo en ratas resulta en atrofia, disminución y pérdida de regeneración hepática ${ }^{(17)}$. En cambio, el tetracloruro de carbono $\left(\mathrm{CCl}_{4}\right)$ se metaboliza activamente por el citocromo $\mathrm{P} 450$ al radical triclorometilo, el cual inicia la lipoperoxidación celular, produciendo daño hepático al comprometer la integridad de las membranas y por la unión covalente de intermedios reactivos a moléculas biológicamente importantes, como el glutatión, induciendo necrosis y daño hepático en 
Tabla 3. Datos de peso corporal obtenido al evaluar el efecto protector de matico sobre la cirrosis inducida en ratas.

\begin{tabular}{|c|c|c|c|c|c|c|}
\hline Variable & Tratamiento & Valor medio & Error estándar & $\begin{array}{l}\text { Porcentaje } \\
\text { variación }\end{array}$ & Valor mínimo & Valor máximo \\
\hline Peso & Normal & 225,1 & 15,8 & 0,0 & 160 & 294 \\
\hline \multirow[t]{6}{*}{$p<0,009$} & Tetracloruro (T) & 218,1 & 18,9 & $-3,1$ & 156 & 284 \\
\hline & $\mathrm{T}+$ Silimarina $25 \mathrm{mg} / \mathrm{kg}$ & 230,6 & 13,0 & 2,4 & 169 & 272 \\
\hline & $\mathrm{T}+$ Extracto $100 \mathrm{mg} / \mathrm{kg}$ & 294,0 & 19,6 & 30,6 & 212 & 366 \\
\hline & $\mathrm{T}+$ Extracto $200 \mathrm{mg} / \mathrm{kg}$ & 245,5 & 16,2 & 9,1 & 194 & 298 \\
\hline & $\mathrm{T}+$ Extracto $400 \mathrm{mg} / \mathrm{kg}$ & 272,6 & 9,6 & 21,1 & 240 & 321 \\
\hline & $\mathrm{T}+$ Fitomedicamento $200 \mathrm{mg} / \mathrm{kg}$ & 255,5 & 12,6 & 13,5 & 194 & 298 \\
\hline
\end{tabular}

Porcentaje $(\%)$ de variación $=[($ Valor medio del tratamiento $X 100) /$ Valor medio del normal $)-100)]$

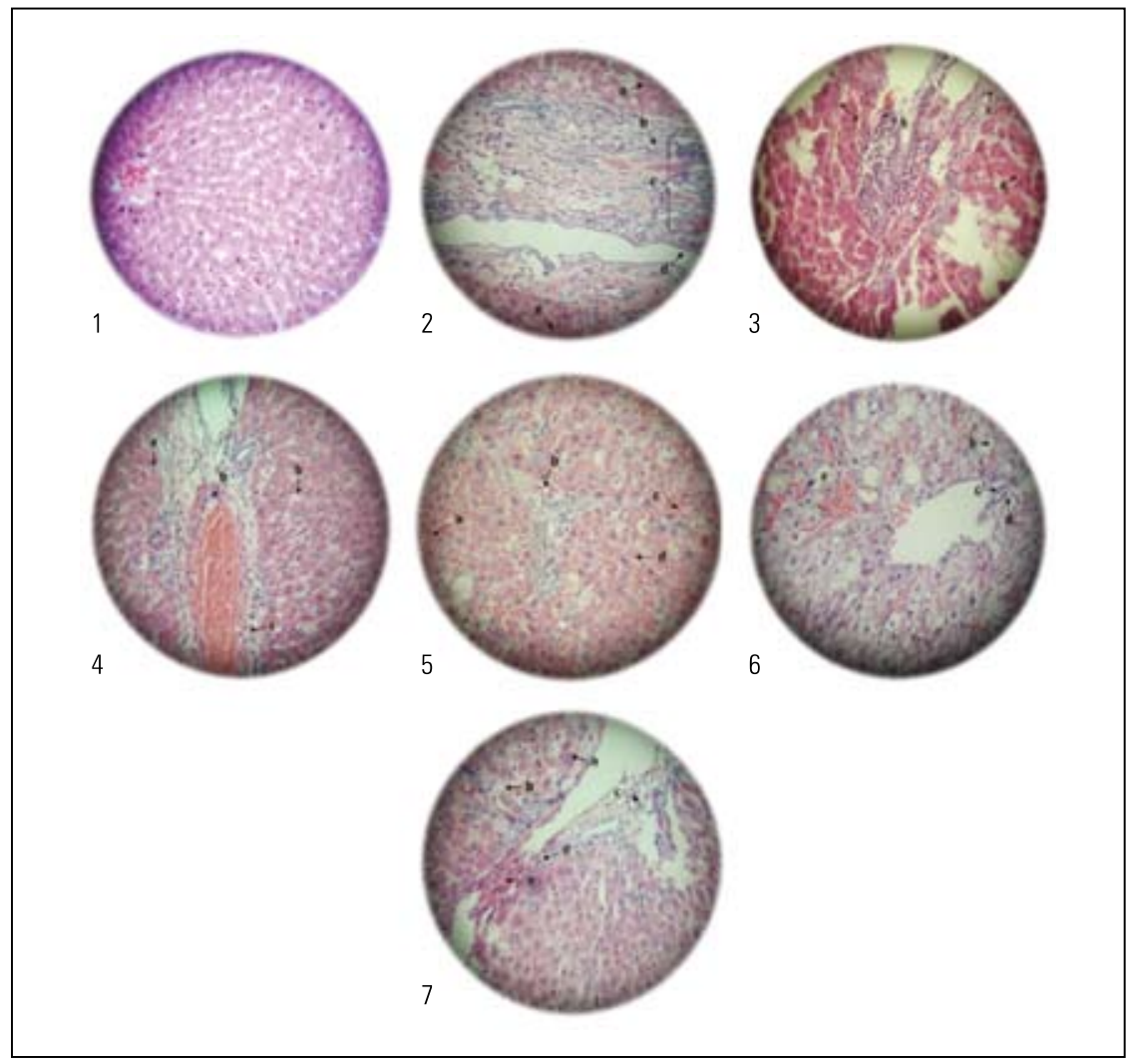

Figura 2. 1. Higado normal; 2. Fenobarbital + tetracloruro de carbono (FTC) (a. microvacuolas, b. Tejido fibroso, c. Bandas cirróticas, d. Espacio de Disse, e. Conductos biliares congestivos); 3. FTC + silimarina $25 \mathrm{mg} / \mathrm{kg}$ (a. Hepatocitos conservados, b. Espacio de Kier con hiperplasia, c. Bandas fibrosas, d. Nódulos de regeneración); 4. FTC + extracto $100 \mathrm{mg} / \mathrm{kg}$ (a. Microvacuolización de hepatocitos, b. Desorganización de conductos biliares y en espacio de Kier, c. Bandas fibrosas); 5. FTC + extracto $200 \mathrm{mg} / \mathrm{kg}$ (a. Nódulos de regeneración, b. Reacción fibrosa,

c. Congestión, d. Desorganización estructural); 6. FTC + extracto $400 \mathrm{mg} / \mathrm{kg}$

(a. Vacuolas grasas, b. Esteatosis, c. Espacio de Kier con discreta alteración,

d. Fibroblastos); 7. FTC + fitomedicamento $200 \mathrm{mg} / \mathrm{kg}$ (a. Microvacuolización discreta, b. Nódulo regeneración, c. Hiperplasia de células de Kupffer, d. Fibroblastos, e. Vasculitis). general ${ }^{(18,19)}$. Por ello, se considera al estrés oxidativo como el principal mecanismo molecular involucrado en la toxicidad por tetracloruro de carbono, el cual tiene rol importante para la inactivación de células Kupffer en la fibrosis hepática inicial inducida por este agente. La producción de radicales libres durante el desarrollo del daño hepático conduce a la disminución de la actividad de la superóxido dismutasa. En ratas con inducción de cirrosis por $\mathrm{CCl}_{4}$, se ha encontrado correlación inversa entre las enzimas antioxidantes y las puntuaciones patológicas y/o de peroxidación lipídica ${ }^{(20,21)}$.

Entre los principales hallazgos histológicos de este estudio estuvo la disminución del daño a la estructura hepática influenciada, por el extracto etanólico de Piper aduncum en ratas con cirrosis inducida por fenobarbital y $\mathrm{CCl}_{4}$ (figura 2). En esta figura se aprecia reducción de daño hepático similar a lo obtenido con la administración de la silimarina; si bien existe daño moderado, la presencia de fibrosis es marcadamente en el control con los inductores y menor en los tratamientos; se desconoce el mecanismo específico por el cual este extracto protege contra el daño hepático; sin embargo, estaría directamente relacionado a los metabolitos que la componen.

Por la tabla 1 se identificó la presencia de metabolitos secundarios, tales como saponinas, alcaloides y compuestos fenólicos, entre taninos y flavo- 
noides, siendo estos últimos los que se encontró en mayor cantidad. Es conocido que la mayoría de los flavonoides son poderosos antioxidantes y poseen diversas actividades antiinflamatorias (el efecto antiinflamatorio del extracto en estudio se evidencia en la figura 1); los flavonoides son inhibidores de la formación de leucotrieno B4, potenciadores de la formación de prostaglandina E2 e inhibidores de la liberación de óxido nítrico ${ }^{(22)}$. Así como, actividad hepatoprotectora relacionada con la capacidad de estos de disminuir el estrés oxidativo y atrapar radicales libres, tanto in vivo como in vitro ${ }^{(23,24)}$; un ejemplo es el caso de la quercetina, el cual ha demostrado ser efectivo contra el daño hepático en ratas con inducción de cirrosis con tetracloruro de carbono, asociado con un incremento de la capacidad antioxidante del hígado para atrapar radicales peroxilo ${ }^{(25)}$; y en ratas con obstrucción biliar crónica, el tratamiento con quercetina resultó en una preservación significativa de la actividad de las enzimas antioxidantes, fibrosis menos pronunciada y marcada inhibición de la proliferación ductular biliar ${ }^{(26,27)}$.

En este estudio, el extracto de P. aduncum se comparó con la silimarina, que es un flavonoide antioxidante aislado del cardo mariano (Silybum marianum (L.) Gaertn) y se utiliza clínicamente como un desintoxicante del hígado y hepatoprotector. Varios estudios recientes han demostrado efectos anticancerígenos de la silimarina ${ }^{(28-30)}$.

Al evaluar diferentes marcadores bioquímicos y hematológicos no hubo diferencia significativa entre los grupos de tratamiento que recibieron tanto el tóxico como la silimarina y las diferentes dosis del extracto de P. aduncum. Sin embargo, sí hubo diferencia en el peso corporal (tabla 3); en los animales cirróticos fue significativamente menor comparado con los animales del grupo control y los otros grupos de tratamiento que recibieron el extracto de $P$. aduncum, mostrando una disminución en la ganancia de peso corporal causado por la administración del tóxico; este efec- to podría estar asociado a alteraciones en la absorción de nutrientes y la utilización metabólica ${ }^{(31)}$.

En la figura 2 se evidencia incremento en la fibrosis, en el colágeno, y por tanto el dañó hepático; la fibrosis hepática es la consecuencia común a lesiones hepáticas crónicas de muchas etiologías. El abuso crónico de alcohol es el principal motivo de que aparezca fibrosis hepática, lo que conduce a la cirrosis, una de las principales causas de muerte en todo el mundo. La fibrosis se caracteriza por una acumulación excesiva de proteínas de matriz extracelular (MEC), tal como el colágeno de tipo I (encontrado en humanos), que se encuentran normalmente en la zona pericentral y perisinusoidal del hígado ${ }^{(32)}$. Como se conoce, existen tres tipos de moléculas: a) colágenos; b) glicoproteínas (fibronectina, laminina); y, c) proteoglicanos ${ }^{(33,34)}$. En la fibrosis hepática destacan por su importancia los colágenos de los tipos I y III.

La silimarina ha ratificado su efecto protector sobre el daño inducido en el hígado, como se evidenció en esta investigación. Se conoce que la silimarina es un compuesto de cuatro isómeros flavonolignanos, conocidos como silibina, isosilibina, silidianina y silicristina, cuya fórmula empírica es C25H22O10. El isómero silibina es el componente mayor y más activo, representando entre 60 y $70 \%$ del compuesto, seguido por silicristina $(20 \%)$, silidianina $(10 \%)$ e isosilibina (5\%) ${ }^{(35)}$. Silipida es un compuesto de fosfatidilcolina de silibina el que asegura la biodisponilidad de silibina ${ }^{(36)}$. El tetracloruro de carbono induce incrementos de transaminasas y colágeno entre 4 a 5 veces más que el normal, mientras que la administración de $\mathrm{CCL}_{4}$ más silimarina disminuyó esas elevaciones significativamente, posiblemente al proteger la membrana celular del hepatocito, y así evitaría el desequilibrio colesterol:fosfolípidos y esfingomielina:fosfatidilcolina, y también redujo los niveles de colágeno ${ }^{(37)}$.

En las condiciones experimentales, se ha demostrado que el extracto eta- nólico de las hojas de Piper aduncum (matico) y su fitomedicamento ejercen efecto protector de la cirrosis inducida en ratas, comparativamente al efecto dado por silimarina.

\section{REFERENCIAS BIBLIOGRÁFICAS}

1. Wolf D. Cirrhosis. emedicine [internet] 2010 [consultado 2010 Nov 21]. Disponible en: http://emedicine. medscape.com/article/185856-overview.

2. Bataller R, Brenner DA. Liver fibrosis. J Clin Invest. 2005;115:209-18.

3. Holdsworth D. Piper aduncum. En: Woodley, E. Ed. Medicinal Plants of Papua New Guinea. Part 1. Weikersheim, Germany: Verlag J. Margraf. 1991.

4. Braga FG, Bouzada ML, Fabri RL, Matos M, Moreira FO, Scio E, Coimbra ES. Antileishmanial and antifungal activity of plants used in traditional medicine in Brazil. J Ethnopharmacol. 2007;111:396-402.

5. Moreno MIN, Isla MI, Sampietro Ar, Vattuone MA. Comparison of the free radical-scavenging activity of propolis from several regions of Argentina. J Ethnopharmacol. 2000;71:109-14.

6. Huang B, Ban X, He J, Zeng H, Zhang P, Wang $Y$. Hepatoprotective and antioxidant effects of the methanolic extract from Halenia elliptica. J Ethnopharmacol. 2010a;131:276-81.

7. Jain A, Soni M, Deb L, Jain A, Rout SP, Gupta VB, Krishna KL. Antioxidant and hepatoprotective activity of ethanolic and aqueous extracts of Momordica dioica Roxb. Leaves. J Ethnopharmacol. 2008;115:61-6.

8. Yi-Hang W, Bing-Jie H, Shen E, Xiao-Meng Z, Yu Z. Regulatory effect of Laggera alata extract on immune mediated liver injury. J Medicinal Plants Res. 2011;5(12):2494-8.

9. Kinoshita S, Inoue Y, Nakama S, Ichiba T, Aniya $Y$. Antioxidant and hepatoprotective actions of medicinal herb, Terminalia catappa L. from Okinawa Island and its tannin corilagin. Phytomed. 2007;14(11):755-62.

10. Arellano P. El libro verde. Ministerio de Salud. 1992:10-1.

11. Lock O. Investigación fítoquímica; Métodos en el estudio de productos naturales. Fondo Editorial Pontificia Universidad Católica del Perú. Segunda Edición. 1994:55-8.

12. Fauli T. Tratado de Farmacia Galénica, Primera Edición. España: Editorial Luzán 5. 1993:45-8.

13. Samudram P, Rajeshwari H, Vasuki R, Geetha A, Sathiya P. Hepatoprotective activity of $\mathrm{Bi}$ - herbal ethanolic extract on $\mathrm{CCl} 4$ induced hepatic damage in rats. Afr J Biochem Res. 2008;2(2):61-5.

14. Buege JA, Aust SD. Microsomal lipid peroxidation. Methods Enzymol. 1978;52:302-10.

15. Troncoso T, Guija E. Efecto antioxidante y hepatoprotector del Petroselinum sativum (perejil) en ratas, con intoxicación hepática inducida por paracetamol. An Fac med. 2007;68(4):333-43.

16. Clemente O, Barajas SM, Serrano MI, Pérez de Ayala MV, Menchén P, Senent MC, et al. Infecciones bacterianas en la cirrosis hepática. Gastroenterol Hepatol. 1986;9:285-90.

17. Hashimoto M, Kothary PC, Raper SE. Phenobarbital in comparison with carbon tetrachloride and phenobarbital-induced cirrhosis in rat liver regeneration. $J$ Surg Res. 1999;81(2):164-9.

18. Wang GS, Eriksson LC, Xia L, Olsson J, Stål P. Dietary iron overload inhibits carbon tetrachloride- 
induced promotion in chemical hepatocarcinogenesis: effects on cell proliferation, apoptosis, and antioxidation. J Hepatol. 1999;30(4):689-98.

19. Aldaba-Muruato LR, Moreno MG, Shibayama M, Tsutsumi V, Muriel P. Protective effects of allopurinol against acute liver damage and cirrhosis induced by carbon tetrachloride: Modulation of NF-KB, cytokine production and oxidative stress. Biochim Biophys Acta. 2011. Disponible en: http://www.sciencedirect. com/science/article/pii/S030441651100239X.

20. Wang L, Cheng D, Wang H, Di L, Zhou X, Xu T, Yang $X$, Liu Y. The hepatoprotective and antifibrotic effects of Saururus chinensis against carbon tetrachloride induced hepatic fibrosis in rats. J Ethnopharmacol. 2009;126(3):487-91.

21. Galisteo M, Suárez A, Montilla MP, Torres MI, Gil A, Navarro MC. Protective effects of Rosmarinus tomentosus ethanol extract on thioacetamideinduced liver cirrhosis in rats. Phytomedicine. 2006;13(1-2):101-8.

22. López-Lirola A, González-Reimers E, Martín Olivera R, Santolaria-Fernández F, Galindo-Martín L, AbreuGonzález P, González-Hernández T, ValladaresParrilla F. Protein deficiency and muscle damage in carbon tetrachloride induced liver cirrhosis. Food Chem Toxicol. 2003;41(12):1789-97.

23. Kim KH, Kim YH, Lee KR. Isolation of quinic acid derivatives and flavonoids from the aerial parts of Lactuca indica L. and their hepatoprotective activity in vitro. Bioorg Med Chem Lett. 2007;17(24):673943.

24. Perez Gutierrez RM, Anaya Sosa I, Hoyo Vadillo C, Victoria TC. Effect of flavonoids from Prosthechea michuacana on carbon tetrachloride induced acute hepatotoxicity in mice. Pharm Biol. 2011;49(11):1121-7.

25. Kviecinski MR, Felipe KB, Correia JF, Ferreira EA, Rossi MH, de Moura Gatti F, Filho DW, Pedrosa RC. Brazilian Bidens pilosa Linné yields fraction containing quercetin-derived flavonoid with free radical scavenger activity and hepatoprotective effects. Libyan J Med. 2011;6 . doi: 10.3402/ljm. v6i0.5651

26. Pavanato A, Tuñón MJ, Sánchez-Campos S, Marroni CA, Llesuy S, González-Gallego J, Marroni $\mathrm{N}$. Effects of quercetin on liver damage in rats with carbon tetrachloride-induced cirrhosis. Dig Dis Sci. 2003;48(4):824-9.

27. Peres W, Tuñón MJ, Collado PS, Herrmann S, Marroni N, González-Gallego J. The flavonoid quercetin ameliorates liver damage in rats with biliary obstruction. J Hepatol. 2000;33(5):742-50.

28. Polyak SJ, Morishima C, Lohmann V, Pal S, Lee DY, Liu Y, Graf TN, Oberlies NH. Identification of hepatoprotective flavonolignans from silymarin. Proc Natl Acad Sci U S A. 2010;107(13):5995-9.

29. Jiang C, Agarwal R, Lü J. Anti-angiogenic potentia of a cancer chemopreventive flavonoid antioxidant, silymarin: inhibition of key attributes of vascular endothelial cells and angiogenic cytokine secretion by cancer epithelial cells. Biochem Biophys Res Commun. 2000;276(1):371-8.

30. Cho YK, Yun JW, Park JH, Kim HJ, Park DI, Sohn Cl Jeon WK, Kim BI, Jin W, Kwon YH, Shin MK, Yoo TM, Kang JH, Park CS. Deleterious effects of silymarin on the expression of genes controlling endothelial nitric oxide synthase activity in carbon tetrachloridetreated rat livers. Life Sci. 2009;85(7-8):281-90.

31. Galisteo M, Suárez A, Montilla MP, Torres MI, Gi A, Navarro MC. Protective effects of Rosmarinus tomentosus ethanol extract on thioacetamideinduced liver cirrhosis in rats. Phytomedicine. 2006;13(1-2):101-8

32. Vera M, Nieto N. Células estrelladas hepáticas y hepatopatía alcohólica. Rev Esp Enferm Dig. 2006; 98(9): 674-684.

33. Greenwel P, Geerts A, Ogata I, Solís-Herruzo JA, Rojkind M. Liver fibrosis. En: Arias IM, Boyer JL, Fausto N , Jakoby WB, Schachter D, Shafritz DA, editors. The Liver Biology and Pathology. 3rd ed New York: Raven Press Ltd.; 1994. p. 1367-81.
34. Greenwel P, Rojkind M. The extracellular matrix of the liver. En: Arias IM, Boyer JL, Chisari FV, Fausto N, Schachter D, Shafritz DA, editors. The Liver: Biology and Pathobiology. 4th ed. Philadelphia: Lippincott Williams \& Wilkins; 2001:469-73.

35. Saller R, Meier R, Brignoli R. The use of silymarin in the treatment of liver diseases. Drugs. 2001; 61:2035-63.

36. Vailati A, Aristia L, Sozze E, Milani F, Inglese V, Galenda P, et al. Randomized open study of the dose-effect relationship of a short course of IdB 1016 in patients with viral or alcoholic hepatitis. Fitoterapia. 1993;64:219-31.

37. Pradhan SC, Girish C. Hepatoprotective herbal drug, silymarin from experimental pharmacology to clinical medicine. Indian J Med Res. 2006;124(5):491504.

38. Khanna SD, Purid D. The hepatotoxic effects of dimethylnitrosamine in the rat. J Pathol Bacteriol. 1966;1:605-8.

\section{Agradecimientos:}

Al Fondo para la Innovación, la Ciencia y la Tecnologia (FINCYT).

Al Instituto Quimioterápico (IQFARMA).

Artículo recibido el 2 de marzo de 2012 y aceptado para publicación el 11 de mayo de 2012.

\section{Conflictos de intereses:}

Los autores expresan no tener algún conflicto de interés.

Correspondencia:

Dr. Jorge Arroyo Acevedo

Facultad de Medicina

Correo-e: jorgeluis_arroyoacevedo@yahoo.es 